\title{
Development of a Biomimetic Robotic Bear: or Is a Bare Bear Bearable?
}

\author{
School of Design, Communication an IT \\ University of Newcastle \\ Callaghan, NSW 2308, Australia \\ michael.dickinson@newcastle.edu.au
}

\author{
Peter Turner \\ School of Electrical Engineering and Computer Science \\ University of Newcastle \\ Callaghan, NSW 2308, Australia \\ peter.turner@newcastle.edu.au
}

\begin{abstract}
This paper presents the issues encountered in the development of a robot based on the biometric influence of a bear. One of the main aims of this research was to explore the possibilities of a robot, which could move between the different styles of motion. The bear offers a unique example of an animal with high mass and bulk, which can move between being a quadruped and a biped.

Our earlier research had explored the development of a robotic dog. A quadruped robot design, suitable for use as a player in the RoboCup Four Legged League [1], which used the parameters of the existing Sony Aibo [2] robot as a starting point. The outcomes of this research have been discussed in papers by Chalup [3] and Lawrence [4].

The current research has extended the previous platform development and reset the objective to a robot with both bipedal and quadrupedal motion possibilities. The original objectives of developing a high quality design with enhanced research programming possibilities, which also coveys a positive and engaging image of Science and Engineering through its form, were maintained. This is specially so, when considering the robot's ability to create interest in the general public, who will view the robot from a perspective outside of discipline specific interests.

The introduction presents the biological inspiration for the current design, including the preparation and material production considerations. This is followed by a discussion of specific features of the robotic bear design, which has been given the name HyKim, followed by a conclusion.
\end{abstract}

Index Terms - Robotic, Biometric, Bear.

\section{INTRODUCTION}

The form of a bear was the inspiration for a robotic platform which is functional in both bipedal and quadrupedal modes. The mechatronic issues of HyKim's design has used the biomimetic propulsion solutions exhibited in real bears in general, but evolved to focus on the polar bear as the primary model. Detailed analysis of the programming capacities of HyKim is available in the paper by Turner [5]. This paper focuses on the design of specific features that make the general public identify the robot as a bear robot.

\section{A. Biologically Inspired Mechanisms and Form}

The biological evolution of large mass mammals like bears which can still remain agile and can obtain bipedal as well as quadruped motion were investigated. They appeared a suitable biomimetic model to explore. Bears have evolved unique characteristics to remain dexterous structures despite their large bulk and mass. To create the required movements in the robotic mechanisms, motors of set sizes need to be used to achieve the desired motion. These motors, batteries and associated circuitry quickly become bulky and heavy.

On the basis of an analysis of the bear's underlying bone structure the biological features, mechanical principles and mechanisms of control provided the starting point for the conceptualization of the robotic bear design. The existing research into biological bear function has been insightful and enhanced the creation of a biped, quadruped cross over robot.

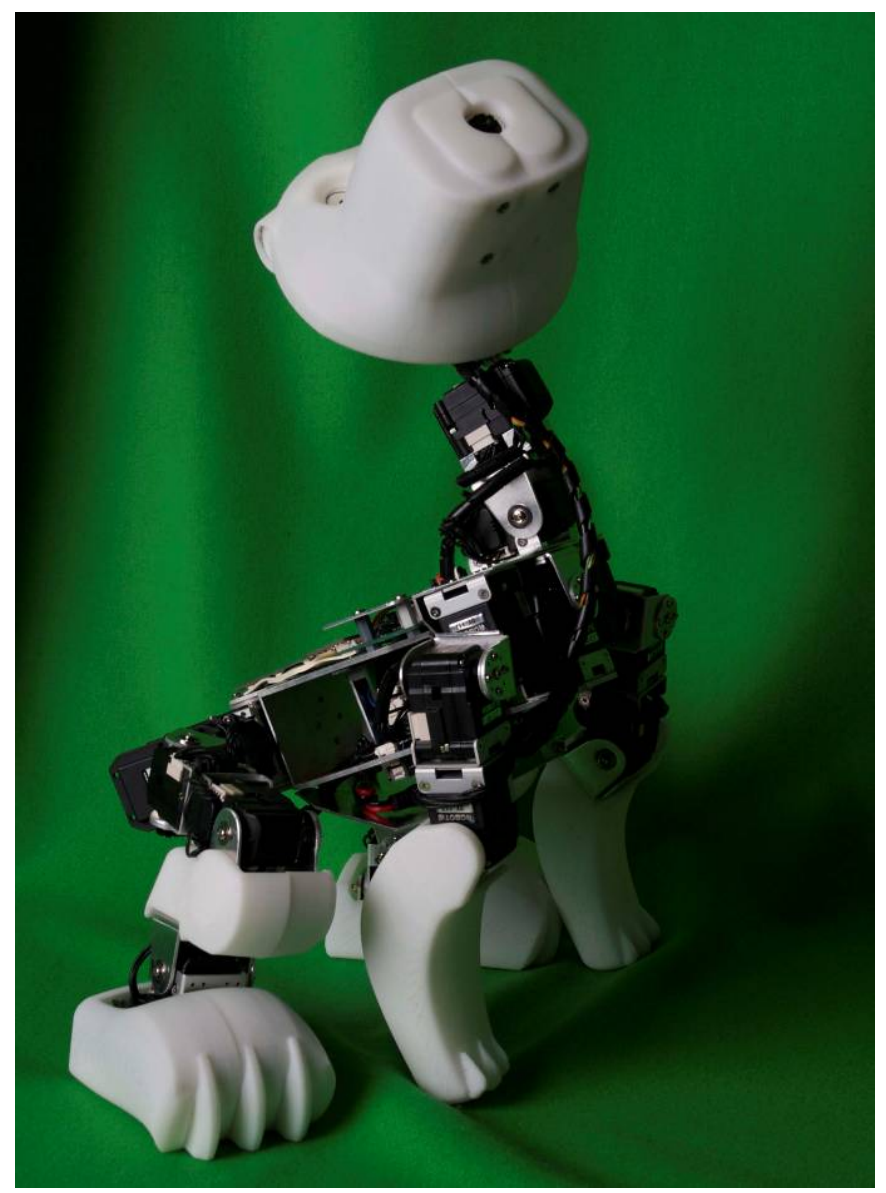

Fig. 1 An image of HyKim at a mid-development stage, with legs and head before the addition of body pieces. Note: the front legs do not have articulated paws at this stage. 


\section{B. Preparation}

At the conceptual stage there were two things to consider. One, what are the biomimetic and aesthetic aspects of bears? And two; at what point have we captured the feel of a bear robot rather than just a robot?

To answer these questions the analysis of the bear has concentrated on those parts of the living animal which structure its function and influence its outward form. The layout of muscle and bone structure provide the principal understanding of the biomechanical mechanisms used by the bear for motion, but the fur and folds of the skin also play a major role in our visual recognition of a bear's bear-ness.

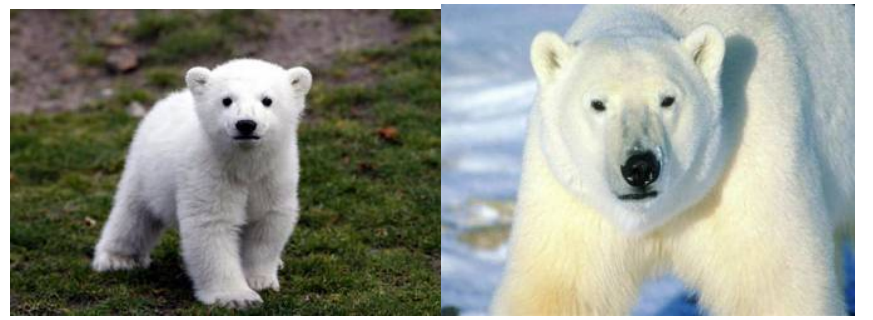

Fig. 2 A variety of bear images were collected which ranged from caricatures to motion studies. They also included the different types of bears from sun bears to grizzlies. Because of the diversity of bear types within the genus it was decided to concentrate on the polar bear to maintain a set focus biomimetically. Images above became the primary visual references [6][7].
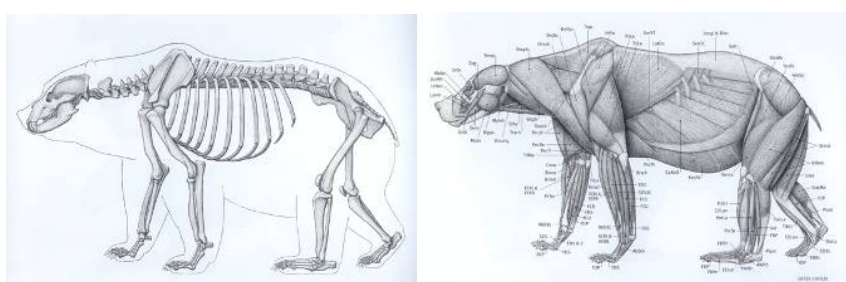

Fig. 3 Left image, rendering of the skeleton of a Brown bear, Ursus arctos, with it's silhouette outline penciled in around the bone structure. Right image, same bear with the muscle structure made visible. Note the difference between the muscle silhouette in image right and the silhouette traced with the pencil outline, image left. Reflects the addition of fat deposits and fur bulk especially in the neck and abdomen areas [8].

An anatomical study of the bears' bone structure reveals that the primary difference between the anatomy of a bear in comparison to a dog or a cat is the bear's plantigrade back foot. It has more in common with a human foot in that it is relatively short and is designed to achieve full contact with the ground from the calcaneum or heel through to the toes. The five fully developed toes are set on an even plain with nonretractable claws. The rear feet are set straight ahead but the front paws are angled inwards slightly in a pigeon toed fashion.

It was also noted that in common with cats, the scapula is raised above the dorsal spines creating the characteristic hump at the shoulders. Despite having no clavicle (collarbone in humans or wish bone in birds) the bear has remarkably flexible limb articulation. The different bear types have remarkably similar bone structures, physical abilities and forms of motion. Differences are often subtle. To maintain consistency as the design developed the polar bear was selected for detailed analysis.

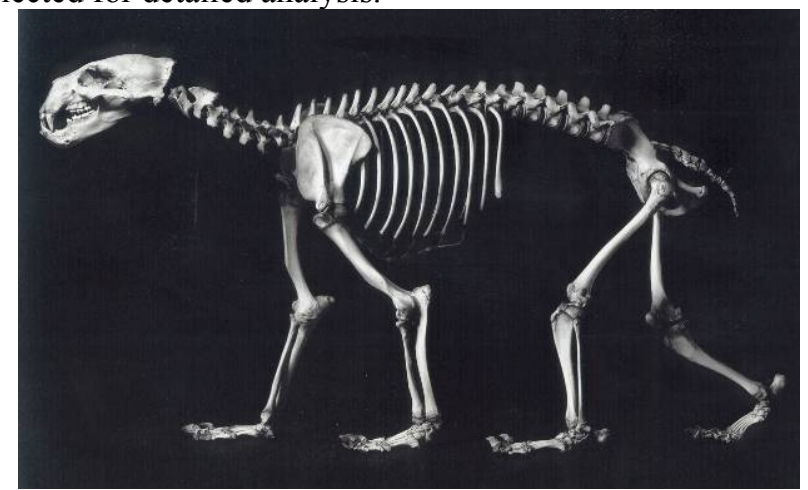

Fig. 4 Polar bear, Ursus martimus, skeleton shoulder height is $85 \mathrm{~cm}$ [9]. Note, the front paws in this skeletal assembly show the fully developed foot structure of the bear. The positioning is atypical of actual use. Refer to Fig 3, which displays the paw use action more accurately.

The polar bear has larger nostrils than other bears, because it needs to preheat the cold air, which enters its lungs. This is an advantage when making disproportionately large holes in the robot head to facilitate the mounting of sensors as unobtrusively as possible. Though the skeletons of all the large bears are very similar, the differences in silhouettes are largely differences in fur bulk and underlying location of fat distribution. The polar bear is regarded as a sub species of brown bears. They can interbreed producing fertile offspring, but they do not mix naturally because of their geographical distribution. The polar bear has adapted to an extreme cold environment and a predominantly carnivorous diet.

A detailed analysis of the polar bears' anatomical structure and motion patterns lead to drawings which transferred the proportions and key pivot points into schematics for the robot design. The details on brown and sun bears in particular were also sourced and used in reference to the polar bears' details to establish working parameters. Suitable biomimetics to create the mechanics that would infer the bear' semantic were thus obtained.

\section{Bear-ness}

The semantics of bear-ness is important. It can be seen in Fig 5., which presents 'the uncanny valley principle', Mori [10], in respect to the depiction of humans. There is a point where the general public rejects the presentation of a human likeness. The use of this principle can be seen in highly successful films like 'The Incredibles' [11] but also in movies where non-humans are depicted like 'A Bugs Life' [12]. The non-realistic depiction of the characters has been a deliberate intentional move to avoid the classic financial failure and the general public's rejection of the technically perfect representation of animated humans in films like 'Final Fantasy' [13]. In the effort to create life like excellence in animation the representation is rejected as being corpse like or zombie-ish rather than creating acceptance and empathy. The authors argue that the same principle can be applied in the development of robotics which uses biomimetic analysis of animals. 


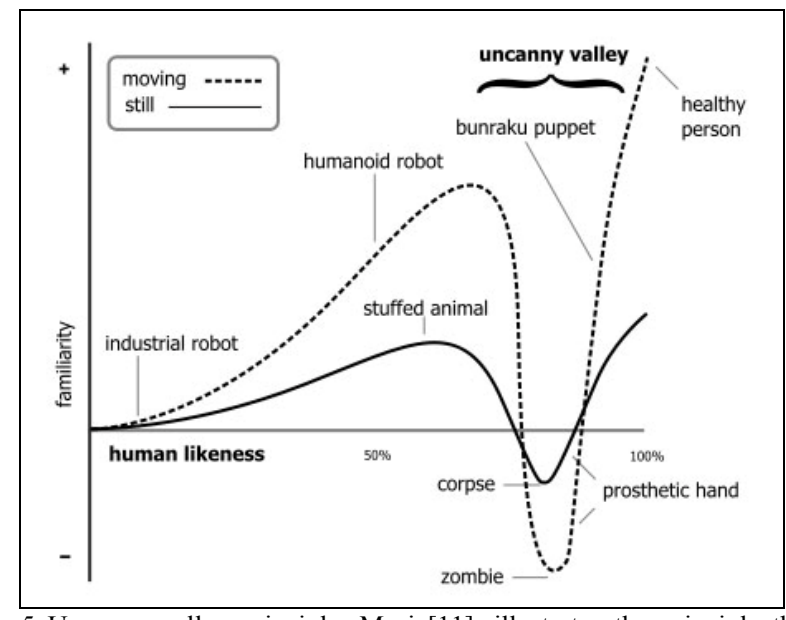

Fig. 5 Uncanny valley principle, Mori [11], illustrates the principle that a sharp decline in the acceptance of a robot is experienced as it moves closer to mimicking life likeness.

With the preparatory analysis and Mori's principle [11] in mind, a basic layout of internal mechanisms of the bear was developed and assembled, Fig 6. Drawings which transferred the proportions of a polar bear's biomimetic details to a proposed mechanical layout were produced and used as references.

It was decided to add a pivot point in the hip area. It is argued that this will be a major advantage in the balance and walking actions of the new bear design. It opens up interesting programming possibilities but from the position of creating a bear aesthetic it created a segmentation of the bear body into separate parts. Significant free space or voids had to be built into the design to allow the desired movement. The segmentation occurred in areas of the bear where there would normally be a smooth transition of fur which is one of the more distinctive features which creates the bulky /cuddly persona associated with bear-ness.

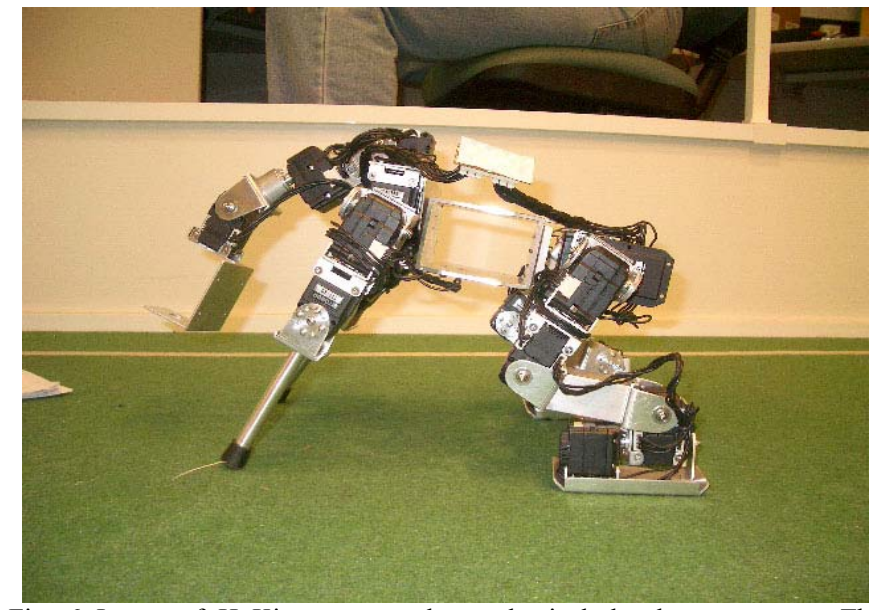

Fig. 6 Image of HyKim at an early mechanical development stage. The aesthetic development of the outer covering of the internal components was run concurrently

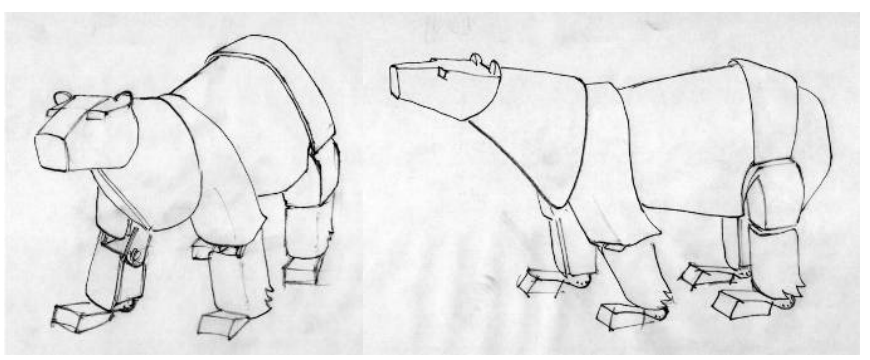

Fig. 7 Examples of bear body development sketches

At this point we had in principle a basic structure and form to proceed with.

\section{Design for printing}

Initially the body parts were envisaged as a series of molded parts made from different grades of urethane. As the design developed and the desire to create a robot, which not only followed the biomimetic form of a bear but also embraced the external look and feel of bear-ness. The advantages of using a 3D printing process were explored and the advantages became evident. It allowed us to make rapid alteration to the form as the internal mechanical and circuitry design changed during the development. To substitute the making of urethane body parts with the $3 \mathrm{D}$ printing process. It became a matter of strengthening the printed parts so they could act functionally not just decoratively.

Inherent to the nature of the ABS 3D printing process selected, the mechanical strength of the layered ABS is less than what would normally be expected, in for example an injection molded ABS component. Specific data on the strength loss was inconsistent. The supplier indicated a $70 \%$ retention of strength, but the material produced through the layered printing process exhibited an effectively 'balsa wood type' of fragility, particularly in thin sections along the 'grain' formed in the deposition of the layers. This had to be allowed for in the design, especially when points of attachment were made mechanically.

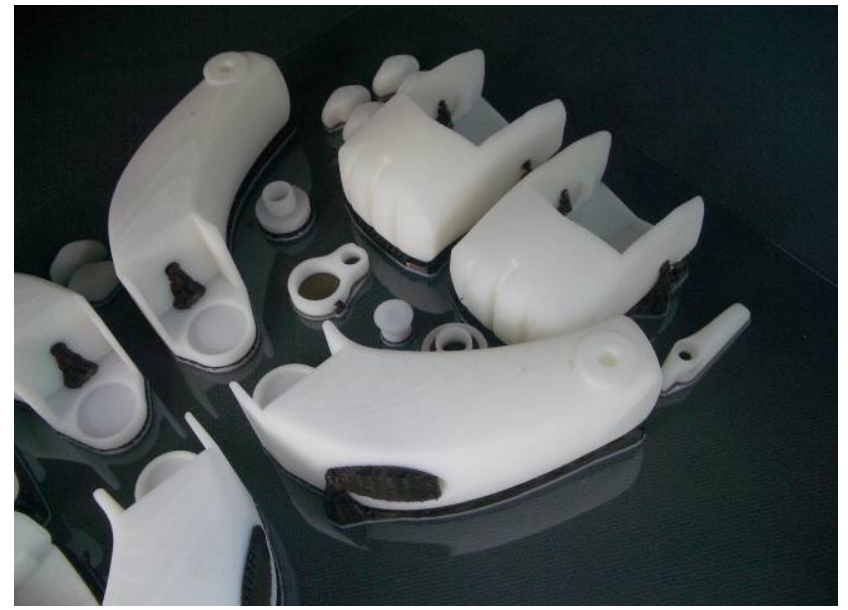

Fig. 8 Examples of ABS plastic bear parts as they could out of the 3D printer. 


\section{SPECIFIC FEATURES}

\section{A. Head design}

The pointed muzzle of the polar bear is more pronounced than in its relative the brown bear, which has developed molars more suitable for the grinding of nuts and berries and consequently has a more square jaw structure.

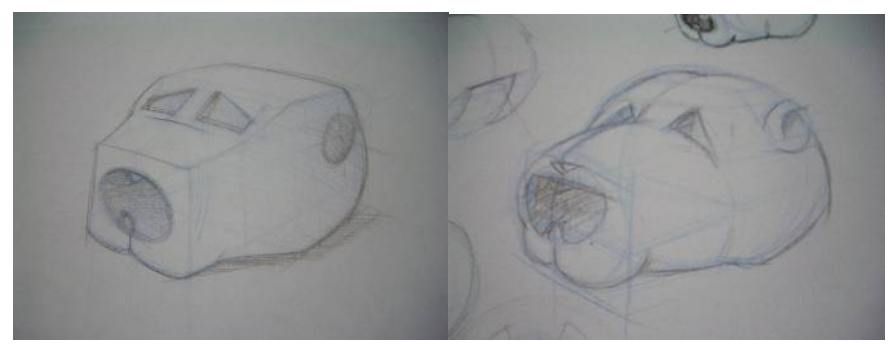

Fig. 9 Examples of different early head design explorations.

Other distinguishing features of the polar bear are its small close-set eyes and rounded ears, though the ears where not an issue apart from something that indicted their presence and added to the bear resemblance. The eyes had to serve a dual function. While still effectively presenting the bears' character, they had to be maximized to allow as large a useable data screen area as possible, without disturbing the bear aesthetic.

In studies by Yarbus, [14] the eyes are clearly identified as the focal feature initially looked at when a person is looking to determine identity. Therefore, the position, shape and size of the eyes and the character presented by the eyes, are not to be dismissed as a minor detail.

The head itself consists of two plastic half shells, which provide a housing for the electronic systems. A detailed discussion of the internal systems housed inside the head can be found in a paper by Turner [5]. These electronic systems allow digital data and distance measurement to be undertaken and had to be located within two parameters, functional effectiveness and aesthetics. The infrared distance sensor was located in the nostril openings, while the digital camera was located mid snout creating a visual triangle with the nostrils. This triangular area could be made a dark colour, probably

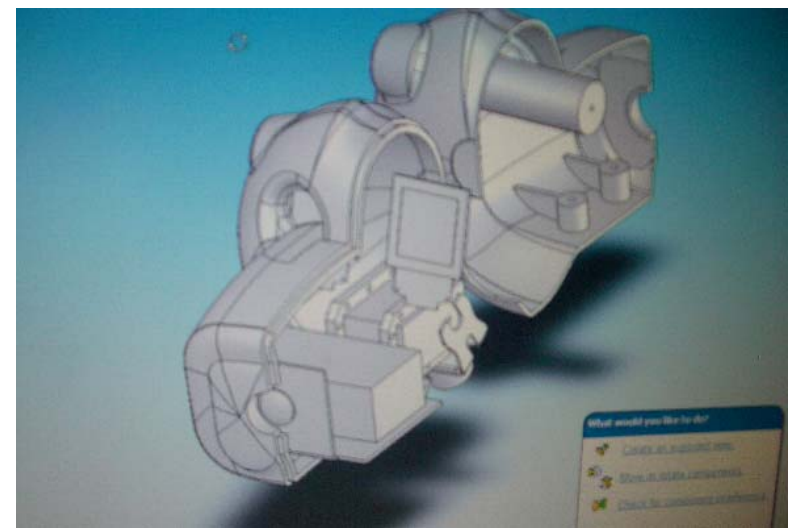

Fig. 10 Example of mid-development drawing of the head where the position and location of internal components is being tested. black, which would further disguise the electronic component while creating the nose shape typical of a bear.

The head assembly is designed so that one half of the head can be removed. This allows access to the internal components, while still fixed in their operating positions to the half of the head that remains attached to the body. This is illustrated in Fig. 11.

Determining the general head size was a combination of remaining within proportion to the general body size and arranging the circuitry and motor to fit. The addition of screens in the eye spaces, to allow digital affects and robot/operator messaging to be explored, proved to be the most difficult component to position. This was resolved by setting the screen's flat surface in a recessed hole, which allowed the outer surface of the eye socket area to be more expressive with the use of complex more organic surface curves.

The aesthetic dimension of the head was addressed by finding a geometric rendition of the basic features of a bear which suited the drawing program. Solid Works was used to create the 3D modeling. Mindful of the need to keep a robotic aesthetic rather than pursuing a lifelike approximation, the argument for steering clear of an exact replication has been argued by Mori [1] in his uncanny valley principle.

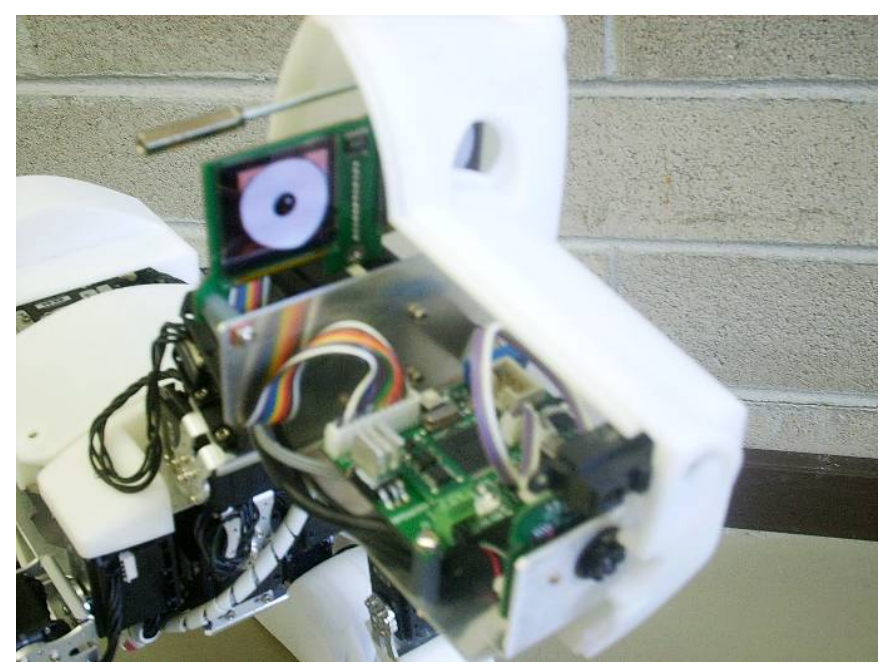

Fig. 11 Image of the head with internal component positioned and mounted in the head half attached to the body

\section{B. Body design}

The body of the robot bear contains the major electronic components and the batteries all of which need at least elementary protection from inadvertent damage. There are also the normal safety issues for the people handling a mid sized piece of electronic equipment. The pieces that make up the body perform this function but are aesthetically under developed at this stage. Because of the pivot point at the hip and shoulder areas the body is segmented. Until future motion studies are conducted and the extent of motion required is established the body parts will remain underdeveloped beyond an essentially functional form. There is also the question of moving more of the internal components weight, for example, the batteries behind the hip into HyKim's rear cavity which 
could lower the center of gravity and help balance the transition from four footed to two footed motion.

\section{Back leg design}

There are two important issues to point out in the back leg design. One is the knee joint. It had to be configured to allow a low centre of gravity when the bear executes a pre-standing posture. In Fig. 6 it can be seen that the bottom of the leg has reached it's maximum ability to fold up into the thigh section of the leg. While staying with commercially available motor bracketing, the section at the knee joint was reconfigured to allow a complete folding of the upper and lower leg. This was very functional but presented as a somewhat cumbersome looking arrangement and has been covered so as to reveal the motors, but soften the angles of attachment.

The second issue was the rear foot itself. To obtain subtlety of balance in the bipedal motion a motor that could manipulate the angle of the foot's relation to the walking surface was included. This resulted in a foot that is disproportionate to both the head and the body. Giving the foot character has alleviated this. Drawing attention to the claws and providing curves where possible to soften the blocky, bulky nature of the internal components.

The cartooning convention of using three fingers on human animations has been transferred to the presentation of three claws despite bears actually having five claws.

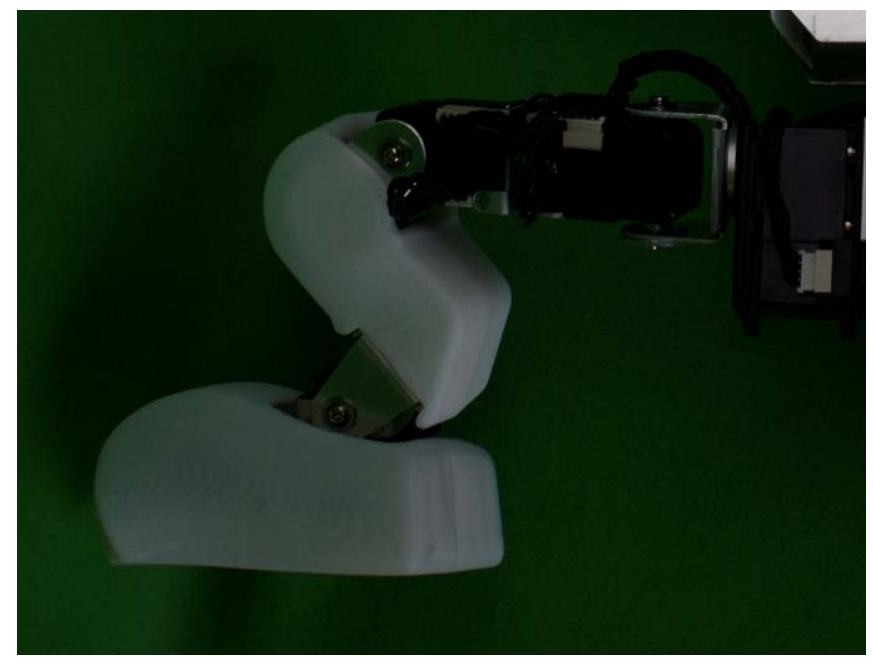

Fig. 12 Image of knee joint and foot design of rear foot.

\section{Front leg design}

Despite the polar bear having the fully developed bone structure in the front feet they use the toe section of each foot predominantly when standing or in motion, refer to Fig3. This is in contrast to the full contact achieved in the rear feet. In the real polar bear there are five fully developed toes that are evenly distributed with non-retractable claws.

In the early development of the front legs a stylized front leg combination paw was tested. It had the practical advantage of part reduction, an example of which can be seen in Fig 1. because of the disproportionate size of the rear feet, which house the large internal motors, the size and shape of the front feet had to not only be functional mechanically, but also visually balance the bear.

The addition of a separate paw component to the front leg was explored for visual balance. The motorizing of the front paw was considered, but was deemed not only an unnecessary additional expense but it would add considerable extra weight at highly leveraged points during the transfer from being a quadruped to a biped and also be visually heavy.

By studying the biomimetic positioning of the paw action in real bears a simple mechanism which is sprung to allow a suitable right angle positioning of the paw when the bear is standing on all four legs, was achieved. When the paw is lifted it snaps back to a profile in line with the general drooped paw position a bear maintains when on two legs. The spring action also provides some level of cushioning as the paw makes contact with the ground. This was an additional benefit in this low cost, low-tech solution to the front paw design.

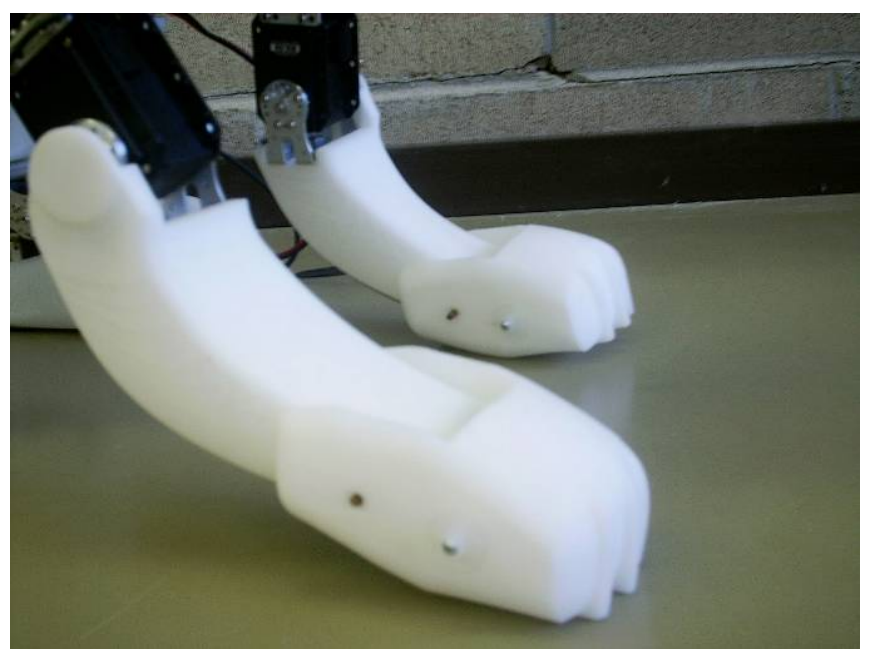

Fig. 13 Image of the front paws. The foot at the front is flexing down as the foot is raised.

\section{Fur}

Despite the polar bear being a large, strong and muscular creature one of the distinctive characteristics of bears is they present relatively little muscular definition, because of the way their fur hangs from the body. One of the directions explored in the development of the robot bear was the application of a fur body suit. Opinion has been divided in whether this is a good option to pursue visually. To date it has literally been an even fifty / fifty split when asking large groups of students. It has also been quite polarizing with people either loving it or hating it. Apart from a straightforward visual aesthetic position, there is one major advantage and one major disadvantage in adding a fur body suit. These two positions will be presented.

The advantage is that in areas like knees and shoulder joints which are complicated to cover and were left looking mechanical because of the added bulk that would be created in attempts to cover such details. A fur covering would smooth 
out these areas in a more bear like aesthetic. This situation also applies to the neck area and the pivot points at the shoulders and hip. So applying a fur body suit presents a way of achieving a form which mimics the soft formless nature of a bear body and conceals areas of the body difficult to cover economically in other ways. It also makes the robot soft and cuddly and draws an immediate reaction of empathy from those who like soft toys.

The major disadvantage is that it is the nature of fur to keep things warm. This is not advantageous to the running of electronics and batteries. A secondary disadvantage is the loss of visual feedback on exact limb positioning when programmers are experimenting with the development of motion.

At present because of the divided opinion the fur suit has been provided as an optional extra rather than a fundamental piece of the bear. But so far people who want the bear have also wanted the suit as well. Whether the fur option is to be used is yet to be tested. At public events, where the purpose is to reach a wider audience than those who have a specific fascination in the cleverness of the internal mechanics and programming, a fur suit may be ideal. Your opinion here will be gratefully appreciated and this area will be the subject of further research.

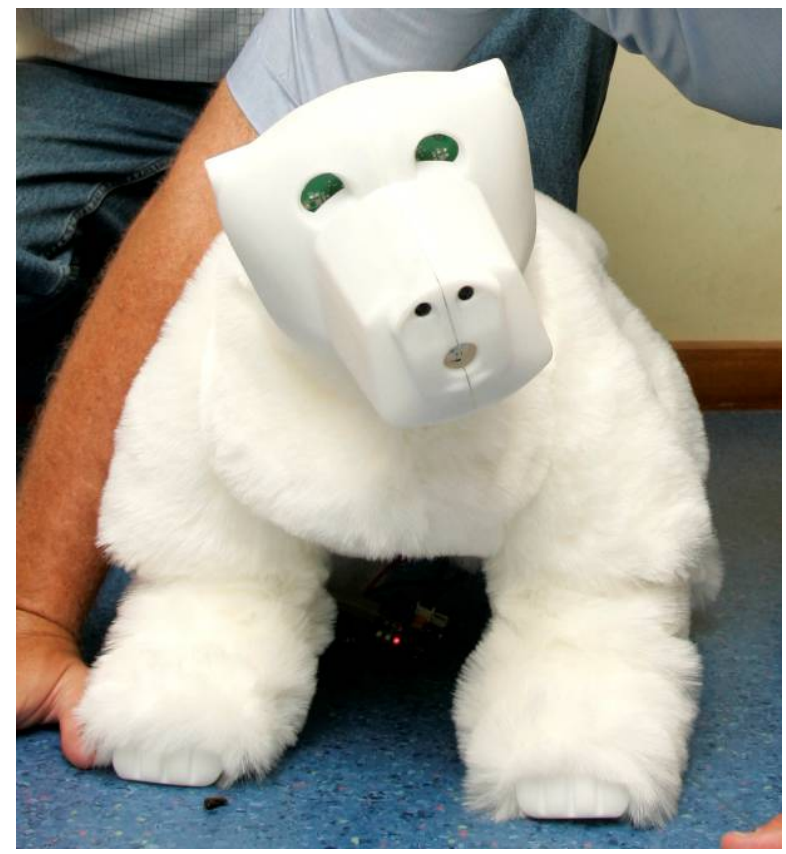

Fig. 14 Image of the current fur suit design. The head has been scaled up to be in proportion with the body but has been left furless intentionally. To bring the aesthetic of the bear robot back to that of a robot which is bear like and away from the feel of a stuffed toy.

\section{CONCLUSION}

A robotic bear following biomimetic principles has been prototyped with self-contained motion control. The design and construction of major features has been discussed.

The process of designing, building and testing the robotic bear has revealed the balancing act required between mechanical/electronic imperatives and the production of an aesthetically desirable robot that invites empathy and interaction. A robotic bear that not only functions as an independently programmable robot but can also present an engaging nature through the design of its external features, has been the objective.

Further research will concentrate on obtaining effective quantitative data which can direct the future development and refine the existing prototype, particularly in respect to the adoption or discarding of the fur.

\section{ACKNOWLEDGMENT}

The authors would like to express their sincere thanks to Daniel Sneddon and Sam Weise for their help in the development of the prototypes. Thanks are also due to all the technicians in the different departments within the University of Newcastle, without their assistance the realization of the robotic bear would not have been possible.

\section{REFERENCES}

[1] Standard Platform League (SPL) organization, URL: http://www.tzi.de/spl/bin/view/Website/WebHome

[2] Sony company, URL: http://www.sony.net

[3] Chalup, S.K., Dickinson, M., Middleton, R.H., Quinlan, M.J., and Turner, P. "Development of a new standard platform for the four legged league of robocup", Multi-Robot Systems: Perception, Behaviours, Learning, and Action, Dagstuhl Seminar No06251, Jun. 2006.

[4] Lawrence, C. Dickinson, M. and Chalup, S. "Designing charm: Harnessing the affective power of form in robotic development", Connected; International conference on Design Education, University of New South Wales, Sydney, Australia, 9-12 July 2007, pp189-194.

[5] Turner, P., Dickinson, M., "HyKim-Development of a robotic bear: Bringing the strength and robustness of bear's biomimetic features to a robot." unpublished.

[6] ISHN "Polar bear image" [online] viewed April 16, 2008, available from World Wide web:<http://www.bri.ucla.edu/nha/ishn/ishn2008-prelim.htm

[7] Hutchison, J. "Polar bear image" [online] viewed July 20, 2007, available from World Wide http://www.therugbyblog.co.uk/animal-xv-could-theybeat-the-all-blacks/

[8] Goldfinger, E., Animal anatomy for artists: The elements of form, Oxford University Press. NY, 2004, pp156-7

[9] De Panafieu, J. Evolution In Action, Natural history through spectacular skeletons, Thames \& Hudson, UK, 2007, p85

[10]Mori, M. “Bukimi no tani”. Translated Karl F. MacDorman and Takashi Minato (The Uncanny Valley). Energy, vol7, no. 4, 1970, pp. 33-35.

[11]The Incredibles, Directed by Brad Bird, produced by Pixar Animation Studios for Walt Disney Pictures, Distributor: Buena Vista Home Entertainment, 2005

[12]A Bugs Life Disney, Directed by John Lasseter \& Andrew Stanton produced by Pixar Animation Studios and released by Buena Vista Distribution 1998

[13]Final fantasy: The spirits within, Directored by Hironobu Sakaguchi \& Moto Sakakibara; written by Al Reinert, Jeff Vintar, Published Culver City, Calif.: Columbia TirStar Home Entertainment [distributor], 2001

[14]Yarbus, A.L., Eye movements and vision, Translation Basil Haigh, A.L. Plenum Press New York 1967. 